\title{
Alterations of Radial Artery Compliance in Patients With Congestive Heart Failure
}

\author{
Cristina Giannattasio, MD, PhD, Monica Failla, MD, Maria Luisa Stella, MD, \\ Arduino A. Mangoni, MD, Stefano Carugo, MD, Massimo Pozzi, MD, \\ Guido Grassi, MD, and Giuseppe Mancia, MD
}

\begin{abstract}
Congestive heart failure is accompanied by several hemodynamic alterations. To investigate whether these alterations include reduced arterial compliance, we studied 25 patients (age $57 \pm 2$ years, mean \pm SE) with a mild or severe congestive heart failure based on clinical symptoms (New York Heart Association class II vs III or IV) and on echocardiographic alterations of left ventricular diastolic diameter and ejection fraction. Radial artery diameter and blood pressure were continvously measured by Doppler ultrasonography and a finger pressure device, respectively. Compliance was calculated by the Langewouters formula, and compliance values were derived throughout the systolic-diastolic pressure range. The area under the compliancepressure curve normalized for pulse pressure was used to compare compliance values in the various groups. Data were obtained both in baseline condition and at the release from a 12-minute brachial artery occlusion.
\end{abstract}

A large body of evidence shows that congestive heart failure is accompanied by a constriction of arterioles throughout a large number of vascular districts. ${ }^{1,2}$ It also shows that this condition is accompanied by a venoconstriction. ${ }^{3}$ Both the arteriolar constriction and the venoconstriction are accounted for by an increased plasma or tissue concentration of vasoconstrictor factors (e.g., norepinephrine, epinephrine, angiotensin II, vasopressin, and so forth), ${ }^{4,5}$ although a reduced secretion and/or influence of vasodilator substances ${ }^{6}$ and an alteration of the vascular wall structure also play a role.?

Hayo $\angle$ et a $l^{8}$ have recently reported that flow-mediated radial arterial relaxation is abnormal in patients with a severe congestive heart failure, suggesting that the hemodynamic derangement of this condition includes a reduced arterial distensibility. Whether, and to what extent, arterial compliance is altered in congestive heart failure has never been directly addressed. It is also unknown whether a compliance alteration occurs in severe congestive heart failure only, or in mild congestive heart failure as well. In the present study we addressed these 2 issues.

\section{METHODS}

Subjects: We investigated 39 subjects $(32$ men and 7 women), with an age ranging from 49 to 60 years. Fourteen subjects (age $53 \pm 2$ years, mean \pm SEM) were

\footnotetext{
From the Cattedra di Medicina Interna, Universirù di Milano, and Ospedale S. Gerardo, Monza, Milan, Italy. Manuscript received December 19. 1994; revised manuscript received and accepted Nay 30,1995

Address for reprints: Professor Giuseppe Mancia, MD, Divisione Medicina interna I, Ospedale S. Gerardo dei Tintori, Via Donize:ti 106, 20052, Moriza, Milan, Italy.
}

Fourteen healthy, age-matched subjects served as controls. Compared with the control group, patients with severe congestive heart failure showed a reduction of baseline compliance index $(-48 \%, p<0.01)$. Furthermore, while in control subjects compliance markedly increased offer brachial artery occlusion $1+43 \%, p$ $<0.01$ ), in patients with severe congestive heart failure no increase occurred. No baseline compliance alteration was seen in patients with mild congestive heart failure in whom, however, the postischemic increase in compliance was also significantly blunted $(-50 \%$ vs controls, $p<0.05$ ). Thus, arterial compliance and arterial compliance modulation are impaired in congestive heart failure. Although more marked in severe congestive heart failure, the impoirment is manifest in mild congestive heart failure as well.

(Am J Cardiol 1995;76:381-385)

healthy, normotensive controls. The remaining 25 subjects (age $57 \pm 2$ years) were patients hospitalized in the S. Gerardo Hospital of Monza, Italy, for congestive heart failure due either to idiopathic dilated cardiomyopathy (18 patients) or to coronary artery disease (7 patients). These patients had a cardiothoracic ratio $>0.55$ (chest roentgenogram) and echocardiographic evidence of an increased left ventricular end diastolic diameter and a reduced left ventricular ejection fraction. Patients were excluded from the study if they had (1) a history of arterial hypertension, diabetes mellitus, or renal insufficiency; (2) a history of myocardial infarction in the previous 6 months; (3) clinical or laboratory (echo-Doppler, digital angiography, computed tomography, etc.) evidence of peripheral major atherosclerotic lesions at the carotid and femoral sites; and (4) chronic atrial fibrillation or other clinically relevant arrhythmias. Prior to the study, all congestive heart failure patients were receiving longterm treatment with furosemide, digitalis, angiotensinconverting enzyme (ACE) inhibitors or nitrates in various combinations and doses. To minimize possible direct effects on arterial compliance, however, ACE inhibitors and vasodilators were withdrawn 5 days before the study. ${ }^{9,10}$ The patients were classified, based on the clinical conditions displayed after withdrawal of ACE inhibitors and/or nitrates, as belonging to the New York Heart Association class II ( $n=16$; mild $)$ and III or IV $(n=9$; severe $)$.

All subjects gave their written consent to the procedures employed after being informed of their nature and purpose. The study protocol was approved by the ethics committees of the institutions involved.

Hemodynamic measurements: Arterial compliance was measured in the left radial artery by an A-mode, ultra- 


\begin{tabular}{|c|c|c|c|c|}
\hline Variable & $\begin{array}{l}\text { Controls } \\
(n=1.4)\end{array}$ & $\begin{array}{c}\text { CHF } \\
\{n=25\}\end{array}$ & $\begin{array}{l}\text { Mild CHF } \\
(n=16)\end{array}$ & $\begin{array}{c}\text { Severe CHF } \\
(n=9)\end{array}$ \\
\hline $\begin{array}{l}\text { Systolic blood pressure }(\mathrm{mm} \mathrm{Hg}) \\
\text { Diastolic blood pressure }(\mathrm{mm} \mathrm{Hg}) \\
\text { Heart rate (beats/min) } \\
\text { End-diastolic diameter }(\mathrm{mm}) \\
\text { Ejection fraction }(\%)\end{array}$ & $\begin{array}{l}117 \pm 5 \\
64 \pm 3 \\
66 \pm 4 \\
47 \pm 1 \\
77 \pm 2\end{array}$ & $\begin{aligned} 106 & \pm 4 \\
62 & \pm 3 \\
73 & \pm 3 \\
66 & \pm 2^{*} \\
36 & \pm 2^{*}\end{aligned}$ & $\begin{aligned} 115 & \pm 6 \\
61 & \pm 4 \\
68 & \pm 3 \\
65 & \pm 3^{*} \\
38 & \pm 3^{*}\end{aligned}$ & $\begin{aligned} 102 & \pm 6 \\
62 & \pm 3 \\
81 & \pm 4^{\star \ddagger} \\
68 & \pm 3^{*} \\
30 & \pm 2^{* \dagger}\end{aligned}$ \\
\hline
\end{tabular}

frequency signals were peaked by an electronic tracer, whose displacement allowed evaluation of the vessel diameter at $50 \mathrm{~Hz}$. Continuous (analog) recording of radial artery diameter was coupled with continuous (analog) recording of blood pressure by a finger pressure device (Finapres 2300, Ohmeda, Englewood, Colorado) positioned on the middle finger of the ipsilateral hand and capable of providing blood pressure values similar to the ones taken invasively from the radial

sonic, echo-tracking device (NIUS02, Asulab, Neûchatel, Switzerland, and Capital Medical Service, Paris, France) ${ }^{9}{ }^{911}$ With the subject supine and the arm fixed at the heart level, a $10 \mathrm{mHz}$ probe was positioned over the radial artery, 2 to $4 \mathrm{~cm}$ above the wrist, and oriented perpendicularly to the longitudinal axis of the artery by using the Doppler signal as a guide. Direct contact with the skin and arterial deformation was prevented by using an ultrasonic gel. After switching to A mode, the backscattered echoes from the inner anterior and posterior walls were visualized on a screen and the related radio- artery. ${ }^{12}$ Both signals were stored and digitized for continuous display on the screen. The arterial diameter and blood pressure signals were sent to a computer that was programmed to use the arctangent model of Langewouters formula, ${ }^{13}$ and provide diameter-pressure curves and compliance-pressure curves over the range of blood pressure values obtained by the finger recording. The echo-tracking device resolution was $150 \mu \mathrm{m},{ }^{14}$ while the finger pressure device resolution was $2 \mathrm{~mm} \mathrm{Hg.}{ }^{12}$ Data were collected by a single operator who was unaware of the patients' New York Heart Association classifications

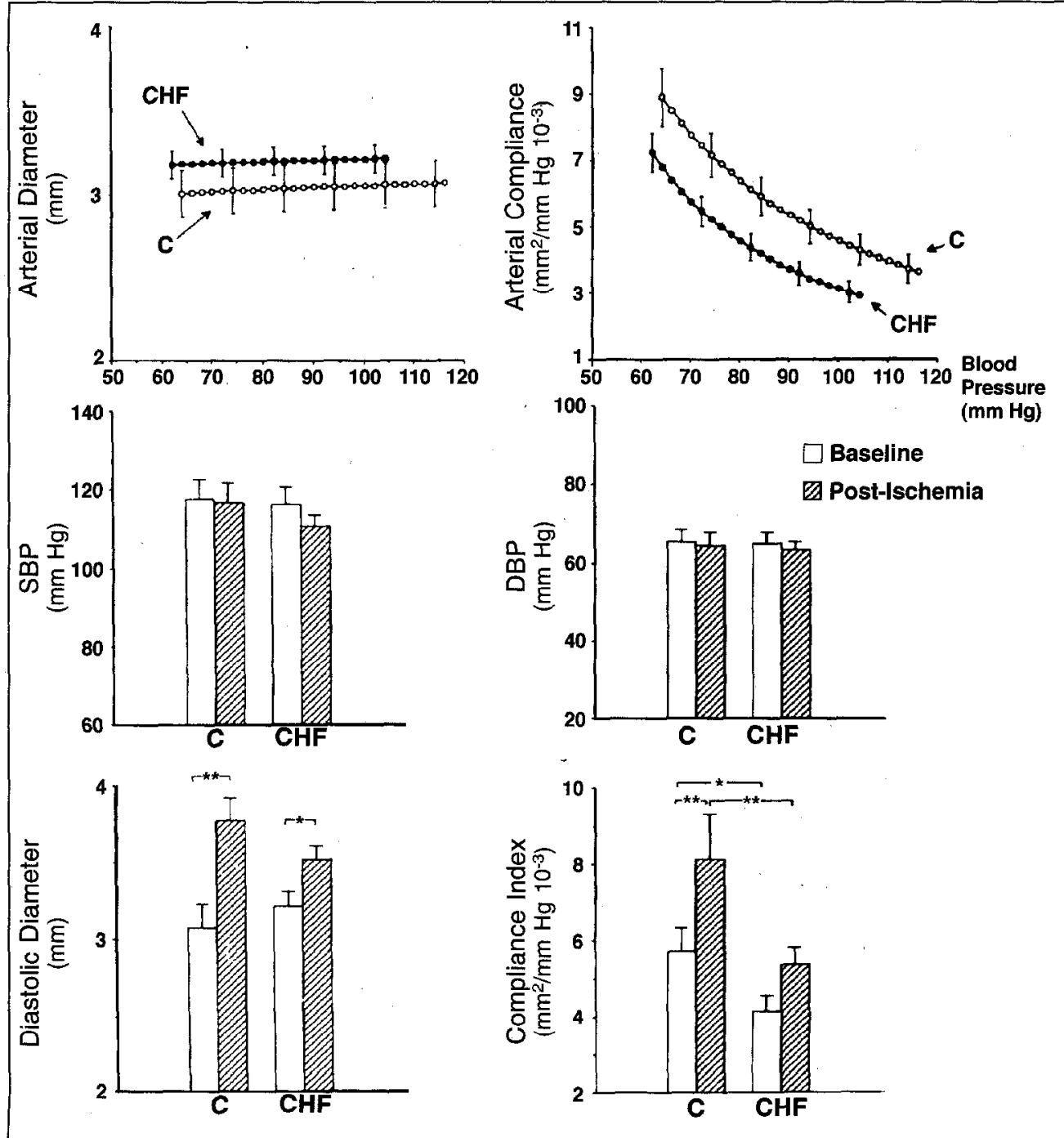

FIGURE 1. Upper panels: Radial artery diameter (leff) and compliance (right) are shown in patients with congestive heart failure (CHF, $n=25$ ) and in control subjects $(C, n=14)$ over the systolic-diastolic pressure range. Lower panels: Systolic blood pressure (SBP) (upper leff) and diastolic blood pressure (DBP) (upper right), and radial artery diameter at diastolic pressure (lower leff) and radial artery compliance index (lower right), are shown in baseline condition and after forearm ischemia for control subjects $(C, n=14)$ and patients with congestive heart failure (CHF, $n=$ 25). Data are shown as mean \pm SEM. ${ }^{*} \mathrm{p}<0.05$; ${ }^{* *} \mathrm{p}<0.01$. 
and echocardiographic data. The intra-operator reproducibility of radial artery diameter measurements was tested in 6 control subjects who were evaluated in 2 different sessions performed at a 3-month interval. As previously reported, the coefficient of variation of the measurements was $4 \% .^{14,15}$ Heart rate values were obtained via the finger pressure signal as the reciprocal of the interval between consecutive systolic beats.

The day before the study, all individuals were subjected to an echocardiogram in $M$ mode, after selection of the measurement section in B mode (Acuson $128 \mathrm{XP} / 5 \mathrm{c}$, Mountain View, California), to obtain left ventricular end-diastolic diameter and ejection fraction. The echocardiographic data were collected by a single operator. The intra-operator reproducibility of end diastolic diameter and ejection fraction measurements, that is, the coefficients of variation of 2 measurements performed by the same operator in 2 different sessions, were $6 \%$ and $10 \%$, respectively.

Protocol and data analysis: All studies were conducted in the afternoon at the S. Gerardo Hospital by using a single arterial compliance measuring device. The protocol was as follows: (1) each subject was placed in the supine position and fitted with the blood pressure measuring and the echo-tracking device, (2) after a 20-minute interval, blood pressure, heart rate, radial artery diameter-pressure curves, and radial artery compliance-pressure curves were measured and stored continuously for 15 minutes, (3) a suprasystolic pressure was applied for 12 minutes to the left arm to occlude the brachial artery, and (4) the hemodynamic measurements were obtained again during the 5 minutes following release of the occlusion. This was done because we and others have shown that radial artery compliance increases markedly after prolonged ischemia, allowing us to study the compliance reserve above baseline. ${ }^{8,16}$ Baseline radial artery diameter-pressure and compliance-pressure curves were obtained from the data collected over the 15-minute period indicated at (2). Postischemic diameter-pressure and compliance-pressure curves were obtained from the data collected over the 15-second period before, and the 15second period after, the maximal increase in diameter was observed (total, 30 seconds). To perform statistical analysis, radial artery diameter and compliance were also expressed as single values. For diameter, this was obtained by averaging the single values corresponding to each single diastolic pressure during (1) 5 periods of 30 seconds taken at 3-minute intervals (baseline), and (2) the 30-second period with the maximal increase in diameter selected after release of brachial artery occlusion. For compliance, it was obtained from the average of the area under the curve relating compliance to arterial blood pressure $(\mathrm{C} \times \mathrm{BP})$ during the 5 periods of 30 seconds during baseline, and during the 30 -second period with the maximal increase in diameter after ischemia. To avoid different area values due to differences in pulse pressure rather than in compliance, the compliance-pressure area was normalized for pulse pressure. The normalized area value for compliance $\left(\left[\mathrm{mm}^{2} / \mathrm{mm} \mathrm{Hg}\right] \times[\mathrm{mm} \mathrm{Hg}]\right) / \mathrm{mm} \mathrm{Hg}$ was referred to as the compliance index.

Individual diameter and compliance-pressure curves were averaged and are shown as mean \pm SEM for each group. Other individual data were also averaged. The statistical significance of the differences in mean values was assessed by the 2-way analysis of variance. The 2tailed $t$ test for unpaired observations was used to indicate the differences between groups, while the 2-tailed $t$ test for paired observations was used to indicate differences between baseline and postischemic periods. The Bonferroni correction was used when comparing more than 2 groups. A p value $<0.05$ was considered statistically significant.

\section{RESULTS}

Table I shows that, compared with control subjects, patients with congestive heart failure had a greater left ventricular end-diastolic diameter and a lower ejection fraction. Heart rate, systolic blood pressure, and diastolic blood pressure were not significantly different in the 2 groups.

In both control subjects and patients with congestive heart failure, radial artery diameter increased slightly but progressively from diastolic to systolic blood pressure (Figure 1). Radial artery compliance showed a concomitant and much more marked decrease. Throughout the systolic-diastolic pressure range, radial artery diameter was similar in control and congestive heart failure patients, while radial artery compliance was less in the latter than in the former group. Radial artery diameter at diastolic pressure was similar in the control and in congestive heart failure group. The congestive heart failure group, however, had a compliance index that was significantly less than that of the control group.

In control subjects, release from prolonged forearm ischemia was associated with no change in blood pressure (Figure 1) and with a marked and significant increase in radial artery diameter and compliance (23\% and $43 \%$, respectively). In contrast, in patients with congestive heart failure, release from prolonged ischemia caused no change in blood pressure, a slight increase in radial artery diameter, and no increase at all in radial artery compliance. As a result of the smaller relative increase in the radial artery compliance index observed in the postischemic period in those with congestive heart failure, as compared with that in control subjects, the difference between the 2 groups became even more evident. Furthermore, a slight reduction in diastolic radial artery diameter also became evident (Figure 2). Table I lists blood pressure, heart rate, and echocardiographic data separately in patients with mild and severe congestive heart failure. Compared with control subjects, the ejection fraction was more clearly reduced in severe than in mild congestive heart failure. End-diastolic diameter was similarly increased in the 2 groups.

Baseline radial artery diameter was unchanged in mild and only slightly reduced in patients with severe congestive heart failure, whereas baseline radial artery compliance was only slightly decreased in mild and markedly reduced in severe congestive heart failure patients. The postischemic increase in compliance was similarly impaired in mild and in severe congestive heart failure patients, while the postischemic increase in diameter was slightly but not significantly reduced in both groups. 
No significant correlation was found between radial artery diameter or compliance and echocardiographic data. An exception was the increase in diameter caused by ischemia, which was inversely related to the ejection fraction values $(r=0.37, p<0.05)$.

\section{DISCUSSION}

Our study shows that radial artery compliance is reduced in congestive heart failure throughout the systolic-diastolic pressure range. It also shows that, whereas in normal subjects, radial artery compliance displays a marked increase after prolonged ischemia, in patients with congestive heart failure, the ischemic-related upward shift of the compliance-pressure curve is blunted. Finally, our observations provide evidence that while in severe congestive heart failure, baseline and postischemic radial artery compliance are both strikingly altered; in mild congestive heart failure, radial artery compliance is normal at baseline but reduced after ischemia, as compared with control subjects. Thus, when assessed in medium-size arteries, compliance is impaired in congestive heart failure. The degree of impairment is related to the severity of the disease, but the phenomenon is an early one, because loss of upward compliance modulation can be seen in mild congestive heart failure as well.

Our study does not investigate the mechanisms responsible for the reduction in radial artery compliance observed in congestive heart failure. It is reasonable to speculate, however, that 3 circulatory abnormalities of congestive heart failure may be involved. One abnormality is the increase in sympathetic drive and plasma or tissue concentration of a number of vasoconstrictor substances, ${ }^{4,5,17-19}$ because this may augment contraction of smooth muscle in the arterial wall, thereby reducing vessel distensibility to the inside pressure. ${ }^{20}$ The second abnormality is the reduction in cardiac contractility and output, because this reduces peripheral blood flow volume and velocity, leading to a reduction of shear endothelium stress ${ }^{2,7}$ and secretion of endothelial relaxing factors that may also result in vessel smooth-muscle contraction, ${ }^{1,6}$ The third abnormality is sodium and water retention, which may cause arterial wall edema and stiffness. $^{21,22}$ It is also reasonable to speculate that, while in more severe congestive heart failure all these abnormalities play a role, in mild congestive heart failure the alteration in arterial compliance is mainly due to the endothelial abnormality, because in mild congestive heart failure sodium and water retention is not a prominent feature. Furthermore, and more significantly, in this condition the alteration in compliance becomes manifest only after release of prolonged blood-flow deprivation, that is, when reactive increase in blood flow is much less than that in normal subjects ${ }^{8}$ and systemic vasoactive agents and sympathetic influences lose their vasomotor effects. ${ }^{23}$

Three other points are worthy of mention. First, in our control subjects, radial artery compliance decreased in a steep, nonlinear fashion from diastolic to systolic blood pressure values. This was also the case in patients with congestive heart failure, indicating that both in normal and in pathophysiological conditions, compliance values have a nonlinear relationship with blood pressure and thus need to be investigated by means of compliance-pressure curves based on a large number of points. ${ }^{.1}$ Second, because in patients with mild congestive heart

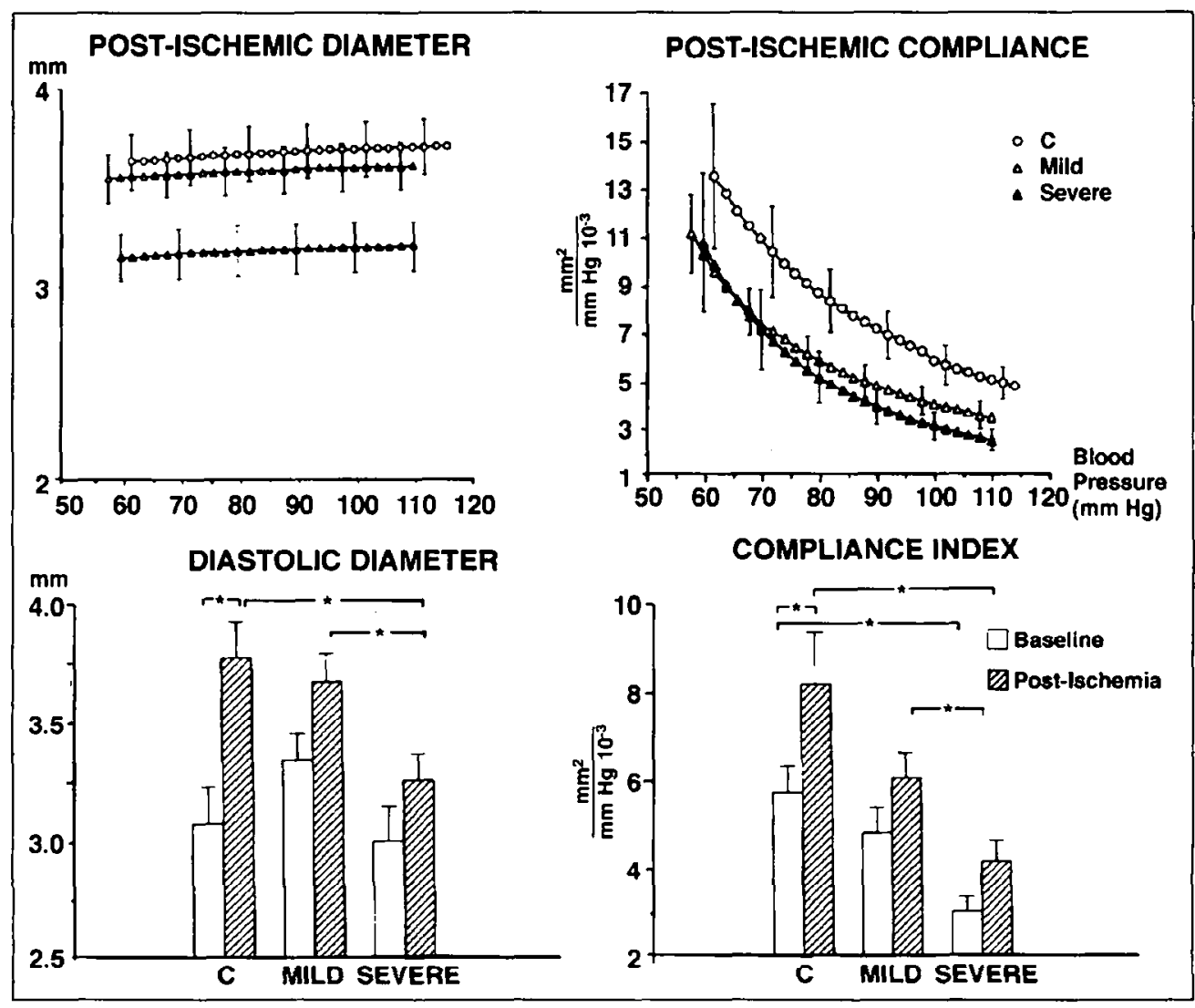

FIGURE 2. Radial artery diameter and compliance of baseline and ofter forearm ischemia. Mean values \pm SEM are shown for control subjects $(n=14)$ and for patients with mild ( $n=16$ ) and severe in $=91$ congestive heort failure. " $p<0.05$. 
failure, radial artery compliance became less than that of control subjects only after a 12 -minute ischemia, prolonged ischemia represents a more sensitive way to uncover arterial compliance abnormalities than do baseline conditions. Moreover, the negative correlation existing between radial artery diameter postischemic increase and ejection fraction suggests that a reduced increase in flow is partially responsible for this alteration, ${ }^{2}$ together with an endothelial impairment. 8,24 Third, our data have pathophysiologic implications. For example, the reduction in arterial compliance leads to an increase in arterial impedance and in cardiac work and oxygen consumption, which may further aggravate the burden of a weakened heart. Furthermore, a reduction in arterial compliance leads to an impaired responsiveness of stretch sensors, such as the arterial baroreceptors, to physiologic pressure stimuli. Because these receptors exert a sympathetic restraint, this may be responsible at least in part for the sympathetic activation characterizing congestive heart failure.

1. Zelis R, Flain SE. Alterations in vasomotor tone in congestive heart failure. Prog Cardiovasc Dis 1982;24:437-459

2. Zelis R, Longhurst J, Capone RJ, Mason DT, A comparison of regional blood flow and oxygen utilization during dynamic forearm exercise in normal subjects and patients with congestive heart failure. Circulation 1974;50:137-143.

3. Muir AL, Nolan J. Modulation of venous tone in heart failure. Am Heart J 1991; 121:148-150.

4. Levine TB, Francis CS, Goldsmith SR, Simon AB, Cohn JN. Activity of the sympathetic nervous system and renin-angiotensin system assessed by plasma hormone levels and their relationships to hemodynamic abnormalities in congestive heart failure. Am J Cardiol 1982:49:1659-1666

5. Goldsmith SR, Francis CS, Cowley AW, Levine TB, Cohn JN. Increased plasma arginine vasopressin levels in patients with congestive heart failure. J Am Coll Cardiol 1983;1:1385-1390.

6. Kubo SH, Rector TS, Bank AJ, Williams RE, Heifeta SM. Endothelium dependent vasodilatation is attenuated in patients with heart failure. Circulation 1991;84: 1589-1596.

7. 7elis R, Mason DT. Diminished forearm arteriolar dilator capacity produced by mineralcorticoid-induced salt retention in man. Implications concerning congestive heart failure and vascular stiffness. Circulation 1970;41:589-592

8. Hayoz D, Drexler H. Mûnzel T, Honnig B, Zeiher A, Just H, Brunner HR, Zelis $R$. Flow mediated arterial dilation is abnornal in congestive heart failure. Circula tion 1993;87(suppI VII):VII-92-VII-96.

9. Perret F, Mooser V, Hayoz D, Tardy Y, Meister JJ, Etienne JD, Farine PA, Marazi A, Burnier M, Nussberger J, Waeber B, Brunner HR. Evaluation of arterial compliance-pressure curves: effect of antihypertensive drugs. Hypertension 1991;18 (suppl 2):77-83.

10. Simon S, Levenson JA, Levy BY; Bouthier JE, Peronneau PP, Safar ME. Effect of nitroglycerin on peripheral large arteries in hypertension. $\mathrm{BrJ}$ Clin Pharmacol $1982 ; 14: 241-246$

11. Tandy Y, Meister IJ, Perret F, Brunner HR, Arditi M. Noninyasive estimate of the mechanical properties of peripheral arteries from ultrasonic and photoplethysmographic measurements. Clin Phys Physiol Meas 1991;3:360-367.

12. Parati G, Casadei R, Groppelli A, Di Rienzo R, Mancia G. Comparison of fin ger intra-arterial blood pressure monitoring at rest and during laboratory testing. Hypertension 1989;13:647-655.

13. Langewouters GJ, Zwart A. Busse R. Wesseling KH. Pressure diameter relationship of segments of human finger arteries. Clin Phys Physiol Meas 1986;7:43-45. 14. Girerd X, Mourad JJ, Acar C. Heudes D, Chiche S, Bruneval P, Mignot JP, Billaud E, Safar M, Laurent S. Noninvasive measurement of medium-sized artery wall thickness in humans: in vitro validation. $J$ Vasc Res 1994;31:114-120. 15. Laurent S, Hayoz D, Trazzi S, Boutouyrie P, Waeber B, Omboni S, Brunne HR, Mancia G, Safar M. Isobaric compliance of the radial artery is increased in patients with essential hypertension. J Hypertens 1993;11:89-98.

16. Giannattasio C, Mangoni AA, Stella ML, Carugo S, Grassi G, Mancia G. Acute effects of smoking on radial artery compliance in humans. $J$ Hypertens 1994;12: $691-696$.

17. Leinbach WN, Wallin GB, Victor RG, Aylward PE, Seindof G, Mark AL. Direct evidence from intraneural recordings for increased central sympathetic outflow in patients with heart failure. Circulation 1986;5:913-919.

18. Thomas JA, Marks BH. Plasma norepinephrine in congestive heart failure. Am $J$ Cardiol 1978;41:233-243.

19. Mancia G, Seravalle G, Giannattasio C, Bossi M, Preti L, Cattaneo BM, Grassi G. Reflex cardiovascular control in congestive heart failure. Am J Cardiol 1992; 69:17G-23G.

20. O'Rourke MF. Arterial Function in Health and Disease. Edinburgh: Churchill Livingstone, 1982.

21. Zelis R, Delea CS, Coleman HN, Mason DT. Arterial sodium content in experimental congestive heart failure. Circulation 1970;41:213-216.

22. Zelis R, Lee G, Mason DT. Influence of experimental oedema on metabolically determined blood flow. Circ Res 1974;34:482-490.

23. Strandell T, Shepherd JT. The effect in humans of increased sympathetic activity on the blood flow to active muscle. Acta Med Scand 1967;182(suppl 472):146-167. 24. Arnold JM, Marchiori GE, Imrie JR, Burton GL, Pflugfelder PW, Kostuk WJ. Large artery function in patients with chronic heart failure. Studies of brachial artery diameter and hemodynamics. Circulation 1991:84:2418-2424 